\title{
Design and Implementation of Base-band Algorithms for OFDM-based Wireless Communication Systerms
}

\author{
Xuncheng Zhu, Zhizhong Ding \\ Department of Communication Engineering \\ Hefei University of Technology \\ Hefei, China \\ zhuxuncheng@gmail.com, zzding@mail.ustc.edu.cn
}

\author{
Oscar Puñal, James Gross \\ UMIC Research Centre \\ RWTH Aachen University \\ Aachen, Germany \\ \{punal|gross\}@umic.rwth-aachen.de
}

\begin{abstract}
Orthogonal frequency-division multiplexing (OFDM) is an appropriate solution to combat inter-symbol interference (ISI) caused by multi-path propagation in wireless communications. OFDM-based wireless communication systems become more and more popular and the base-band algorithm is one of the significant parts of the systems. In this paper, we present the design and implementation of base-band algorithms for OFDM-based wireless communication systems. The results show that the designed algorithms are effective for implementation of the system.
\end{abstract}

Keywords-orthogonol frequency-division multiplexing (OFDM), wireless communication; base-band algorithms

\section{INTRODUCTION}

OFDM has been rapidly utilized for wireless communication standards. This is because OFDM deployments typically protect against ISI by using the guard interval. There are many OFDM-based standards such as digital audio\&video broadcasting, the IEEE $802.11 \mathrm{a} / \mathrm{g} / \mathrm{n}$ standards for wireless local area networks (WLANs), as well as IEEE 802.16 for metropolitan access, worldwide interoperability for microwave access (WiMAX) [1][2][3]. With the development of these standards, OFDM is widely adopted and this is very helpful for the next generation telecommunication networks.

OFDM is a frequency-division multiplexing (FDM) scheme used as a digital multi-carrier modulation method. A serial data stream is divided into several parallel data streams and modulated on the closely-spaced orthogonal sub-carriers. Therefore, OFDM over single-carrier schemes has the ability to cope with severe channel conditions. Despite the advantageous of OFDM-modulation, OFDM has several weaknesses such as high peak-to-average-power ratio (PAPR) and a high sensitivity to Doppler shift and frequency synchronization problems. In practical OFDM-based wireless communication systems, time and frequency synchronization as well as channel estimation need to be solved in the receiver. In recent years, various synchronization algorithms [4], [5] and algorithms of the channel estimation [6] have been proposed. This contributes to the continuing development of the OFDMbased wireless communication systems. It is required for us to establish the basic structure of the base-band OFDM systems so as to implement these good algorithms in the above system.

In this paper, the design and implementation of OFDMbased algorithms for base-band wireless communication systems are presented. In order to test the reliability of the base-band system, 10 sets of random binary data are utilized for data processing on Microsoft Visual $\mathrm{C}++6.0$ software platform by using $\mathrm{C}$ language. The simulation results show that the decoded output data is correct and therefore the designed base-band systems are effective and reliable.

This paper is organized as follows. Section II describes the structure of the base-band system. Processing technology for OFDM-based base-band signals is presented in Section III. Section IV provides the specific implementation of the baseband algorithms. Finally, Section V concludes the paper.

\section{THE STRCTURE OF THE BASE-BAND SYSTEMS}

The block diagram of the base-band systems is shown in Fig. 1.

The transmitted base-band data consist of OFDM symbols and a preamble followed by the symbols. The designed preamble is utilized for the synchronization and channel estimation. Through a rate $1 / 2$ convolutional code [7], channel encoding is realized. In order to protect against error bursts, the coded data is interleaved and mapped to symbols from 16 quadrature amplitude modulation (QAM). The mapped symbols are modulated by OFDM encoder which performs 64point inverse fast Fourier transforms (IFFT). Each OFDM signal consists of the IFFT output symbols and a cyclic prefix (CP). After OFDM modulation, framing is performed. Then the resulting signal is sent to the receiver.

In the receiver, synchronization and channel estimation is initially performed. After $\mathrm{CP}$ removal and OFDMdemodulation with FFT, channel equalization is finished by the equalizer. The demodulated symbols are de-mapped, and then de-interleaved. Finally, Viterbi decoder determines the most probable transmitted bit sequence.

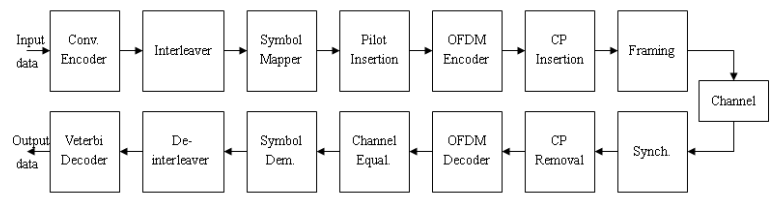

Figure 1. The structure of the base-band system. 


\section{PROCESSING TEChNOLOGY FOR OFDM-BASED BASE- BAND SIGNALS}

Key technology of base-band algorithms include: convolutional encoding and decoding, OFDM modulation and demodulation, synchronization, and channel estimation and equalization. These four algorithms are of great importance to the implementation of the algorithms for the whole base-band systems.

\section{A. Convolutional Encoding and Decoding}

In terms of an $(\mathrm{n}, \mathrm{k}, \mathrm{N})$-convolutional encoder, each $\mathrm{k}$-bit information symbol encoded is transformed into an n-bit symbol, where $\mathrm{k} / \mathrm{n}$ is the code rate. In the convolutional code, encoded n-bit symbol is not only related to the current $\mathrm{k}$-bit symbol, but also to several leading information symbols. Taking a $(2,1,3)$ convolutional encoder for example, its polynomial matrix of a product is given by

$$
G(D)=\left[1+D+D^{2}, 1+D^{2}\right]
$$

where $D$ represents the delay operator. Therefore, it is implemented by a modulo-2 adder. Viterbi algorithms of a convolutional code are based on the maximum likelihood decoding. Its basic principle is add-compare-select operation and finding the sequence which is the closest to the transmitting sequence. According to the different ways of determining the branch metric value, Viterbi decoding is divided into soft-decision and hard-decision decoding. This paper focuses on hard-decision decoding. The algorithm calculates the Hamming distance by comparing the received sequences with all possible symbol path sequences, and then for the same node we have a comparison with the accumulated path metrics and choose the optimal path. Finally, we find the node with the largest accumulate cost, begin trace-back from this node and get decoding output data.

\section{B. OFDM Modulation and Demodulation}

Basic principle of OFDM is that a serial data stream is divided into $\mathrm{N}$ parallel data streams at low symbol rate which are then modulated on $\mathrm{N}$ sub-carriers which are orthogonal to each other and are sent after superposition. The orthogonality allows for efficient demodulator implementation using the FFT algorithm on the receiver side. We consider an OFDM system with $\mathrm{N}$ sub-carriers. The OFDM signal is given by

$$
s(t)=\sum_{k=0}^{N-1} X(k) \exp \left(j 2 \pi f_{k} t\right),
$$

where $X(k)$ is $k$ th data symbol, and $f_{k}$ is the sub-carrier frequency, $s(t)$ is sampled, we denote $t=n / f_{s}, n=0,1,2, \ldots, N-1$. The sampled signal is

$$
s\left(n / f_{s}\right)=\sum_{k=0}^{N-1} X(k) \exp \left(j 2 \pi n f_{k} / f_{s}\right) .
$$

In (3), $f_{s}$ is the sampling frequency, and we denote $f_{k}=k f_{s} / N$. The result is

$$
s\left(n / f_{s}\right)=\sum_{k=0}^{N-1} X(k) \exp (j 2 \pi k n / N) .
$$

$$
=N x(n), n=0,1,2, \ldots, N-1,
$$

Where $X(k)$ represents discrete Fourier transform (DFT) of $x(n)$. From the above mathematical derivation we observe that the modulation of digital base-band OFDM signal can be implemented by IDFT and likewise the demodulation by an implementation of the DFT.

\section{Synchronization}

This paper mainly researches design and implementation of OFDM base-band algorithms, so it ignores the frequency synchronization and analyzes the synchronization from the angle of timing synchronization. Timing synchronization lets the receiver have the same cycle sequences with the transmitter, and finds the beginning and end time of each symbol.

There are four kinds of algorithms of timing synchronization and they are introduced and analyzed as follow.

- The first algorithm is the algorithm of the frame timing synchronization for energy detection based on null symbols. For burst-mode communication systems, if there is no data coming, the received signal is the noise signal. Once the data frame arrives, the data and noise signals are detected in the receiver and the energy will gradually increase. Through the comparison between the energy value of the received signal and the threshold value, we can judge whether the data arrived or not. The major disadvantage of the algorithm is that the threshold value is closely related to the energy of the received signal. Thus, it is difficult for the systems to judge whether data come or not.

- The second algorithm achieves frame timing synchronization with training sequences. There are two identical training sequences while $\mathrm{N}$ sampled symbols are delayed in the first sequence. Through the maximum value of the autocorrelation function between the two sequences, the best timing moment can be found. The implementation of the algorithm is easy and it is effective.

- The third algorithm is the algorithm of the maximum likelihood symbol timing synchronization based on cyclic prefix. In order to eliminate ISI, cyclic prefixes are added in OFDM. Therefore, the information that cyclic prefixes carry can be utilized to find the best position of the timing synchronization. The algorithm uses the correlation between cyclic prefix and its duplicated part to implement timing synchronization. Although this method improves the transmission efficiency compared to the methods based on training sequences and it is easy to realize, the effect of the timing estimation is poor and it is difficult to be directly applied to the practical communication systems.

- The last algorithm is the one of the symbol timing synchronization with training sequences. Since the receiver has accurately known the training sequences, the symbols in the receiver match with the training sequences can be found by using the cross-correlation operation. The algorithm can be easily implemented and the timing is more accurate.

In our implementation, we opt for two different timing synchronization methods. Based on the above discussion, we 
choose the algorithm of the frame timing synchronization with training sequences as the coarse synchronization algorithm and the last algorithm is utilized to calculate the fine synchronization.

The coarse synchronization algorithm is represented as

$$
\begin{gathered}
P(d)=\sum_{m=0}^{L-1} r(d+m) r^{*}(d+m+16) . \\
R(d)=\sum_{m=0}^{L-1} r(d+m+16) r(d+m+16)^{*} . \\
A(d)=\frac{|P(d)|^{2}}{|R(d)|^{2}} .
\end{gathered}
$$

Above, $r(m)$ is the discrete received signal, $d$ is the delayed time, $P(d)$ is the delayed autocorrelation operation, $R(d)$ is the autocorrelation operation, $A(d)$ is the output result of normalized delayed autocorrelation, and $L$ is the length of correlation window.

The fine synchronization algorithm is given by

$$
\begin{gathered}
U(d)=\sum_{m=0}^{M-1} r(d+m) s(m)^{*} . \\
V(d)=\sum_{m=0}^{M-1}|s(m)|^{2} . \\
C(d)=\frac{|U(d)|^{2}}{|V(d)|^{2}} .
\end{gathered}
$$

Here, $s(m)$ is the local copy of the short training sequences, $d$ is the delayed time, $U(d)$ is the cross-correlation operation, $V(d)$ is the autocorrelation operation, $C(d)$ is the output result of the normalized cross-correlation, and $M$ is the length of correlation window.

Through the operations of coarse synchronization and fine synchronization, we can find the accurate position of the timing synchronization, and then correctly realize the demodulation operations.

\section{Channel Estimation and Equlization}

Channel estimation aims at estimating frequency response of wireless channel estimation between the transmitting antenna and the receiving antenna. The values of the frequency response of OFDM systems should be estimated. First of all, the algorithm of time-domain channel estimation with training sequences needs the calculation of the invertible matrix and thus the computation is too complex. Secondly, the algorithm of the frequency-domain channel estimation based on inserted pilot symbols is computationally quite complex while its application also causes a certain amount of signal-to-noise ratio (SNR) loss due to power allocation on the positions of the pilot symbols. Finally, the least squares (LS) algorithm of the frequency-domain channel estimation based on pilot symbols is easy to realize and is used in practical systems.
Therefore, this paper makes use of the LS algorithm of the frequency-domain channel estimation based on pilot symbols to perform channel estimation. The algorithm is easy to implement and doesn't require previously acquired channel knowledge. We consider each sub-carrier of OFDM systems which is $S(k), k=0,1,2, \ldots, N-1$. The time-domain transmitting sequences are given by

$$
s(n)=\frac{1}{N} \sum_{k=0}^{N-1} S(k) \exp \left(j \frac{2 \pi}{N} k n\right), 0 \leq n \leq N-1 .
$$

If the length is longer than the maximum time-delay of channel, the received OFDM sampled data are represented as

$$
r(n)=s(n) * h(n)+w(n), 0 \leq n \leq N-1 .
$$

Here, * denotes convolution, $h(n)$ represents channel frequency response, and $w(n)$ is the additive white Gaussian noise (AWGN) with zero mean and a variance of $\mathrm{E}\left[|w(n)|^{2}\right]$. From (12), FFT transform is denoted by

$$
R(k)=S(k) H(k)+W(k), 0 \leq n \leq N-1,
$$

where $W(k)$ and $H(k)$ represent frequency-domain AWGN and frequency-domain $h(n)$. The result of channel estimation is derived as

$$
\begin{aligned}
H^{\prime}(k)=\frac{R(k)}{S(k)} & =H(k)+\frac{W(k)}{S(k)} . \\
& \approx H(k), 0 \leq n \leq N-1 .
\end{aligned}
$$

Here, $H^{\prime}(k)$ is the estimated value of $H(k)$.

If there are several training sequences, we can utilize the time-domain arithmetic mean method to achieve smooth noise. Hence, this paper uses two equal training sequences so as to realize channel estimation. After OFDM demodulation, we can implement equalization.

\section{IMPLEMENTATION OF BASE-BAND ALGORITHMS}

Through research and analysis of theories and thoughts for key technology of base-band algorithms, we propose the following implementation architecture for a base-band OFDM transmission system. In the following, we will discuss all baseband algorithms from the transmitter and receiver.

\section{A. Transmitter}

Binary data is the input to the base-band system. Implementation steps of the transmitter are given as follows:

- Binary data is sent to a $(2,1,7)$ convolutional encoder, and then the output data of the encoder needs to be interleaved. In the practical process of the implementation of the convolutional encoder, the convolutional state diagram is used to easily achieve the function of the encoder instead of the modulo-2 adder. We make use of the number to represent the state from 1 to 64 . Switch language can be utilized to get the next state and the output two symbols due to the current state and the input symbol "0" or " 1 ". The above output symbols are written into the 32-by-30 rectangular matrix row by row and read out column by column, thus the interleaving operation is achieved. 
- The output data of the interleaving need 16-QAM mapping with switch language in line with the constellation diagram. Every 4 symbols can be transformed into an integer from 1 to 16 , and then get the corresponding mapped values due to the integers. After mapping, the expanded 64 complex numbers consist of 48 complex numbers representing the data, four pilot symbols inserted in the particular place and another twelve zero complex numbers. This is convenient for the 64point IFFT operation.

- $\quad$ OFDM modulation can be implemented by using 64point IFFT algorithm. The cyclic prefixes of the modulated OFDM symbols are inserted where a cyclic prefix consists of the last quarter of an OFDM symbol. Therefore, an OFDM signal includes 80 complex numbers and the total useful data have four OFDM signals.

- $\quad$ Short training sequences, long training sequence and 50 zero complex numbers can serve as the preamble [8]. According to the special data we set, the two sets of data are calculated by using the IFFT algorithm and the sampled data of the output results of the IFFT transform can be obtained. The one set of data are used for the short training sequences and the other for the long training sequences. A frame consists of the useful data and the preamble followed by the data.

\section{B. Receiver}

The whole system chiefly achieves the base-band algorithms, so the transmitted signal is directly sent to the receiver. Implementation steps of the receiver are given as follows:

- Coarse timing synchronization is calculated before the preamble symbols reach the receiver. When the operation begins, the calculated value of the synchronization is zero. The value is greater than zero when the preamble comes, and it will be greater and greater. As the value is normalized, it is not greater than 1 . If the calculated value is smaller than the chosen threshold value, it means that the position of the synchronization is not found and the value of the coarse synchronization needs to be continued to compute, or else fine synchronization demands to be computed. Because the short training sequences have several identical symbols, the correlation can be utilized to calculate the maximum value of the normalized cross-correlation and find the fine timing position through the last maximum value.

- After the coarse timing synchronization and the fine timing synchronization, channel estimation begins to be operated to get the channel response value. For the long training sequences which have two identical symbols, they are demodulated by FFT transform, and the transformed pilot symbols cope with the phase shift. Then divide the mean between the two frequency-domain symbols where each symbol has 48 useful complex numbers by the mean between the two time-domain symbols in the transmitter and the response frequency value can be acquired. When the operation of the channel estimation is over, the useful data should be also demodulated. After OFDM-modulation, pilot symbols are used to deal with the phase shift and then the demodulated data are equalized by the frequency response value. At the same time the equalized data need to be put together for the de-mapping.
- 16-QAM de-mapping needs to be performed next. If it is the smallest value that the Euclidean distance between the data symbol and the one point of the constellation diagram, the data symbol is transformed into the integer, and then corresponds to the binary symbol with switch language.

- De-interleaving operation is opposite to the interleaving operation and the 30 -by-32 rectangular matrix is utilized. Viterbi decoder begins to transform every two symbols into an integer and uses the XOR gate to implement an exclusive disjunction. Through the gate, the Hamming distance is easy to compute. A linked list needs to be created to store the uplink and downlink information and the addresses of the nodes. Every time we should calculate 128 operations of the add-compare-select method and leave 64 sets of accumulated values. Simultaneously, the symbol " 0 " or " 1 " and the address of the node should be noted. Finally, the pointer can be used to realize the operation of the backtracking and find the most possible path sequence.

\section{The Simulation and Analysis of the System}

In this part, the analysis and simulation experiments of the synchronization and channel estimation are given as follows.

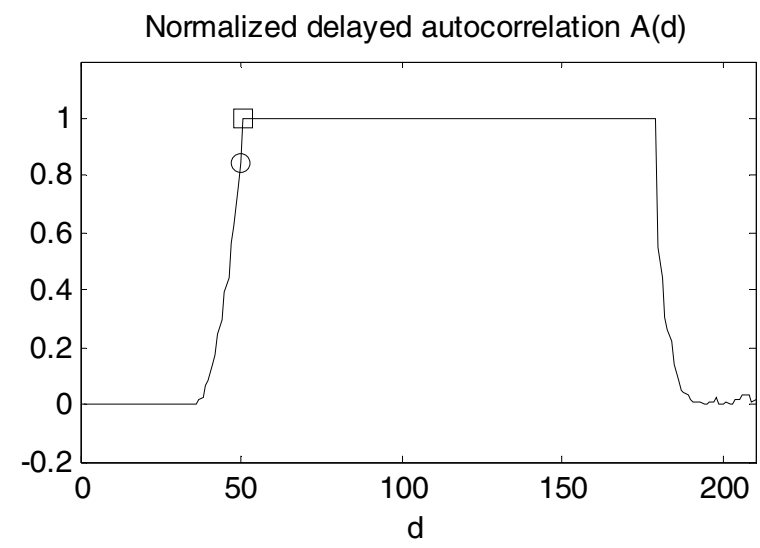

Figure 2. The implementation of the coarse timing sychronization.

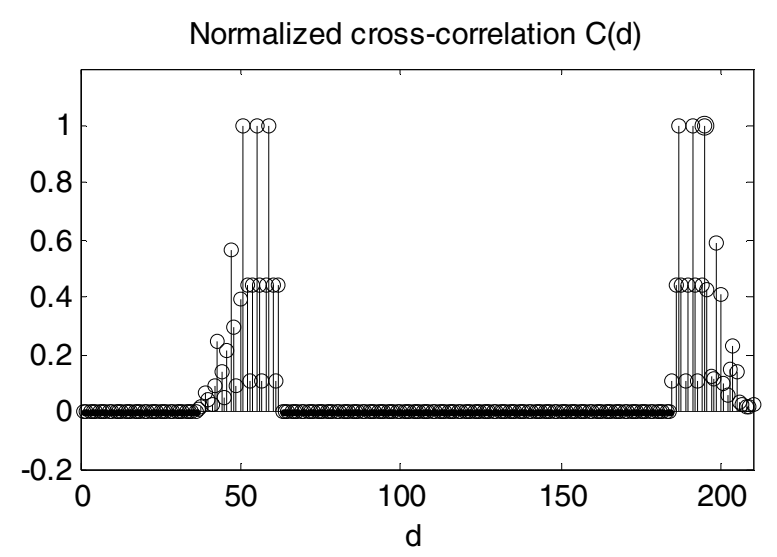

Figure 3. The implementation of the fine timing sychronization.

For the coarse timing synchronization, the simulation experiment is shown in Fig. 2. The value of $A(\mathrm{~d})$ is zero in the 
beginning because there are 50 zero complex numbers in front of the frame. When the frame is coming, the value is greater than 0 and it will gradually increase. In Fig. 2 the "o" represents the value of $A(50)$ that is 0.8403 and the square points to the value of $A(51)$ which is 1.0000 . As the chosen threshold value is 0.9 , the position of the coarse timing synchronization is found and $d$ is equal to 51 .

In order to make it more obvious for the important data in Fig. 3 , the values of the middle data are set to 0 . If the chosen threshold value is smaller than 0.8403 in order to improve the stability of the timing synchronization, the result of the coarse timing synchronization will be wrong. The greatest value of $C(d)$ is 0.5869 except for the maximum value which is 1.0000 . Therefore, 0.8 can serve as the threshold value and it can improve the stability. According to Fig. 3, the "o" points to the value of $C(195)$ which is 1.0000 corresponding to the last maximum value of $C(d)$.

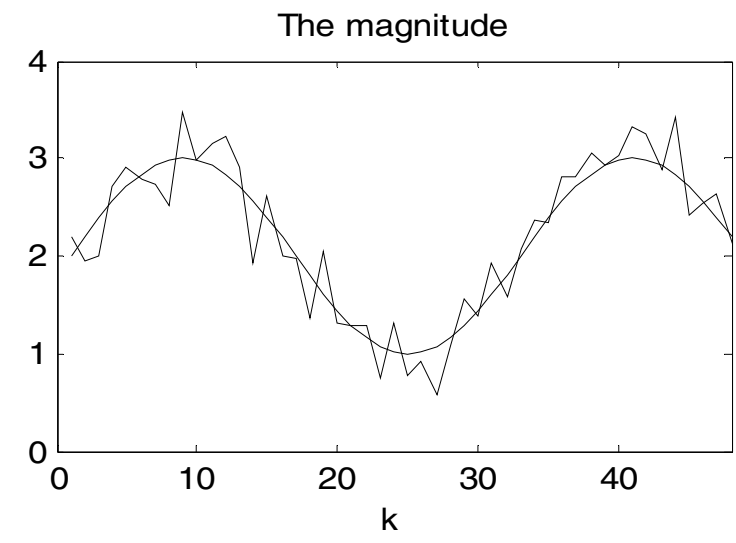

Figure 4. The magnitude of the chanel frequency response.

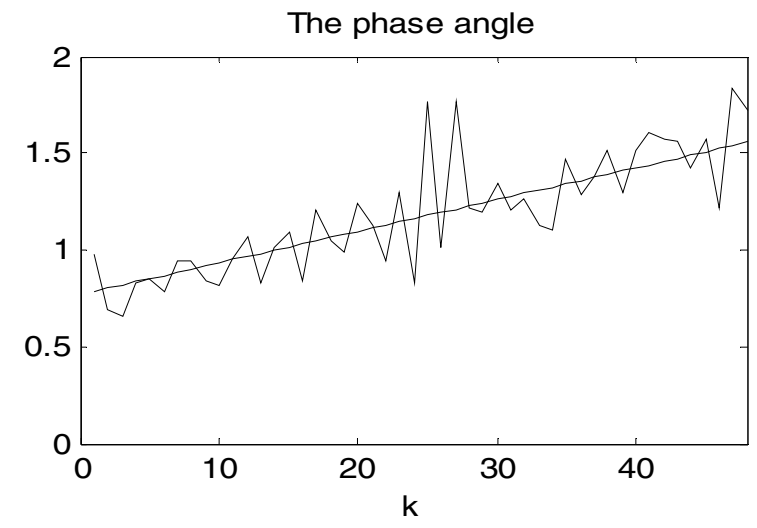

Figure 5. The phase angle of the chanel frequency response.

After the timing synchronization, the transmitted training signals can begin to compute the channel estimation. We make use of 48 subcarriers to take the signal information, so the training 48 complex numbers to perform the operation of the channel estimation. The signal passes through the designed distortion and AWGN channel can be added, and the SNR is $14 \mathrm{~dB}$. The basic outline of the estimated magnitude curve of the channel frequency response is very close to the ideal magnitude curve from Fig. 4. Fig. 5 shows that the estimated phase angle fluctuates according to the ideal curve of the phase angel.

For the above analysis and figures, we can know that the algorithms designed are basically effective. The algorithms of the timing synchronization can find the best position. The operation of channel estimation can help us to get the estimated values of channel frequency response and they are utilized to calculate the channel equalization.

\section{CONCLUSION}

In this paper, the algorithms for OFDM-based wireless communication systems are implemented by using $\mathrm{C}$ language on Microsoft Visual $\mathrm{C}++6.0$ software platform. In order to test the reliability of the base-band systems, 10 sets of random binary data are processed and the output results are correct. It is proved by the simulation results that the systems have good characterizes such as high reliability and effective implementation. Since the algorithms are realized on software platform, hardware platform based on DSP can be considered to implement the designed algorithms as a future work.

\section{REFERENCES}

[1] I. Koffman, et al., "Broadband Wireless Access Solutions Based on OFDM Access in IEEE 802.16," IEEE Comm. Mag., Vol. 40, No. 4, pp. 96-103, April 2002.

[2] B. Li, et al., "A Survey on Mobile WiMax," IEEE Communications Magazine, Vol. 45, No. 12, pp. 70-75, December 2007.

[3] X.D. Yang, et al., "Performance Analysis of the OFDM Scheme in DVB-T," The 6th IEEE Circuits and Systerms Symposium on Emerging Technologies, Vol. 2, No. 32, pp. 489-492, 2004

[4] M. H. Hsieh and C. H. Wei, "A Low-Complexity Frame Synchronization and Frequency Offset Compensation Scheme for OFDM Systems over Fading Channels", IEEE Trans. Veh. Technol., vol. 48, pp. 1596-1609, Sept. 1999.

[5] G. Santella, "A Frequency and symbol synchronization System for OFDM Signals: Architecture and Simulation Results", IEEE Trans. Veh. Technol., vol. 49, pp. 254-275, Jan. 2000.

[6] Y. Mostofi, D. C. Cox, A. Bahai, "Effect of Frame Synchronization Errors on Pilot-aided Chanel Estimation in OFDM: Analysis and Solution," Proc. 5th International Symposium on Wireless Personal Multimedia Communications 2002, vol. 3, pp. 1309-1313, Oct. 2002.

[7] IEEE Standards Department, IEEE Press, “ANSI/IEEE Standard 802.11 Wireless LAN," 2001.

[8] IEEE Std 802.11a-1999, "Wireless LAN Medium Access Control (MAC) and Physical Layer (PHY) specifications: High Speed Physical Layer in the 5 GHz Band," July 1999. 